\title{
Prevalence and Risk Factors of Autism Spectrum Disorders in Children Aged 18-36 Months in Hubei Province, China
}

\author{
Qiong Dai ${ }^{1, \dagger}$, Xuejun Kong ${ }^{2, \dagger}$, Yiqing Song ${ }^{3}$, Aiqin Zhou ${ }^{1}$, Xuan Zhang ${ }^{1}$, Ping Zhang ${ }^{1}$, Minghui Li ${ }^{1}$, \\ Xinglian Liu ${ }^{1}$, Jun Wang ${ }^{4}$, Haiqing $\mathrm{Xu}^{1,}$ \\ ${ }^{1}$ Health Care Department, Maternal and Child Health Hospital of Hubei Province, Huazhong University of Science and Technology, Wuhan, \\ China \\ ${ }^{2}$ Synapse Program/Martinos Center, Massachusetts General Hospital, Harvard Medical School, Bostons, USA \\ ${ }^{3}$ Department of Epidemiology, School of Public Health, University of Indiana, Bloomington, USA \\ ${ }^{4}$ Department of Biomedicine and Biopharmaceuticals, Hubei University of Technology, Wuhan, China
}

\section{Email address:}

daiqiong74@163.com (Qiong Dai), xkong1@mgh.harvard.edu (Xuejun Kong),yiqsong@iu.edu (Yiqing Song), aegean2022@126.com (Aiqin Zhou),xaken@sina.com (Xuan Zhang),361290411@qq.com (Ping Zhang), 1mh701125@126.com(Minghui Li), alian_bb@126.com(Xinglian Liu),jun_wang@hbut.edu.cn(Jun Wang), xuhaiqing9@126.com (Haiqing Xu)

${ }^{*}$ Corresponding author

${ }^{\dagger}$ Qiong Dai and Xuejun Kong are co-first authors.

\section{To cite this article:}

Qiong Dai, Xuejun Kong, Yiqing Song, Aiqin Zhou, Xuan Zhang, Ping Zhang, Minghui Li, Xinglian Liu, Jun Wang, Haiqing Xu. Prevalence and Risk Factors of Autism Spectrum Disorders in Children Aged 18-36 Months in Hubei Province, China. Journal of Family Medicine and Health Care. Vol. 5, No. 4, 2019, pp. 64-69. doi: 10.11648/j.jfmhc.20190504.15

Received: November 6, 2019; Accepted: November 22, 2019; Published: December 6, 2019

\begin{abstract}
We recruited 15,417 participants aged 18 to 36 months in central China using a stratified, multi-stage random sampling method. For children with a positive result in Toddlers screening test, further diagnostic assessment of autism spectrum disorders (ASD) was performed using the childhood autism rating scale and the Diagnostic and Statistical Manual of Mental Disorders. ASD prevalence among these children was $0.58 \%$ in Hubei province. Multivariate-adjusted logistic regression models were then used to explore potential ASD risk factors. One parent aged 35 or older, preterm birth, and birth weight $<2.0 \mathrm{~kg}$ remained significantly associated with elevated ASD risk $(P<0.05)$. These findings indicated that management of adverse perinatal factors may prevent the occurrence of ASD.
\end{abstract}

Keywords: Autism Spectrum Disorders, Prevalence, Risk Factors

\section{Introduction}

Autism spectrum disorders (ASD) are a group of developmental disabilities characterized by impairment in social interaction and communication and featured by restricted, repetitive, and stereotyped patterns of behavior. Symptoms of ASD typically appear before age 3 [1]. Prevalence of ASD has risen sharply over the past two decades [2-5]. Recently, the estimate increased from approximately $1 \%$ in large studies of ascertaining possible ASD cases in the US and the UK to $2.6 \%$ in one South Korean study that actively screened for ASD in a general population [6]. ASD prevalence varied worldwide from 1.4/10,000 children in the Arabian
Peninsula to $185 / 10,000$ children of Asian population [7]. In Europe, the highest prevalence has been observed in Sweden $(115 / 10,000)$, while the lowest is in Croatia $(2-3 / 10,000)$ [8]. The amount and cost of care needed to provide support and services for families with ASD patients have been increased dramatically. ASD has become a major public health problem globally.

The incidence of ASD has been rising due to increased awareness of this disease and advancement of clinical diagnosis [9]. About 25 years ago, ASD was thought to be a rare condition and only occur mostly in western countries. Even today, most people in Africa either didn't know this disorder or considered it as a disease belonging to the western 
developed countries. ASD prevalence and occurrence in China is still unclear and its existence is even under debate.

ASD is a complex multifactorial disease with unknown etiology. Multiple risk factors, including environmental, biologic and genetic factors, were believed to stay associated with development of ASD [10]. Children born from older parents are at a greater risk of having ASD [11]. The critical period for ASD development may occur before, during, and immediately after birth. The studies on prevalence and risk factors of ASD in Chinese children are barely existing, mainly due to lack of epidemiologic research in large population of children.

This population-based study in Hubei Province (central China) was designed to investigate the prevalence of ASD among children aged 18-36 months and to explore potential paternal and perinatal risk factors associated with ASD to provide guidance for early intervention for children with ASD.

\section{Materials and Methods}

\subsection{Participant Recruiting}

Our study was conducted at Hubei Province, which was part of a national survey of disabled children aged 0-6 years in China in 2003. Participants were recruited with a stratified, multi-stage random sampling method.

First, 29 districts and counties in urban or rural areas were randomly selected from total 89 districts and counties in Hubei province. At each district or county, two communities were randomly selected. All the children aged 18 to 36 months from the selected sites were included in our study. Children with the following conditions were excluded from the study: (1) with severe physical diseases (i.e. cancer, hearing impairment, aphasia, severe physical disability, and seizure disorders), (2) with psychiatric diseases (i.e. phobia and obsessive-compulsive disorder), and (3) with severe intellectual disability. In total, 1,242 children were excluded, including 414 children with serious physical diseases, 362 children with a family history of mental or psychiatric diseases, and 466 children with severe intelligent disorders or cognitive problems.

\subsection{Methods}

Two pediatricians from one local hospital in each of the selected sites got trained at Hubei Maternity and Child Health Care Hospital for the sampling procedure, as well as the appropriate use of screening and diagnostic tools for ASD. These trained pediatricians conducted the screening test of ASD by face-to-face interviews with 15,417 children and their parents in local hospitals and collected baseline information through questionnaires. Each questionnaire includes parental age, education level, occupation, income, place of residence, gestational age, birth weight, infection during pregnancy, fetus protection therapy, perinatal asphyxia, pathologic jaundice, hypoxic-ischemic encephalopathy and gestational diabetes.

For the child with the positive result for the Checklist of Autism in Toddlers (Chat-23) test, further assessment for a confirmed diagnosis of ASD was carried out by two senior pediatricians in the Department of Child Health Care in Hubei Maternity and Child Health Care Hospital. The childhood autism rating scale (CARS) together with the Diagnostic and Statistical Manual of Mental Disorders, Fifth Edition (DSM-5) was used for ASD diagnosis. Diagnostic criteria include persistent deficits in social communication and social interactions across multiple contexts. ASD severity was defined based on social communication impairment and restricted, repetitive patterns of behaviors.

\subsection{Statistical Analysis}

Continuous variables were expressed as mean \pm standard deviation. Categorical variables were presented as number. Student t-test was used to compare the difference between ASD children and non-ASD controls for each continuous variable. Chi-square test was applied to measure the difference between children with and without ASD for each categorical variable. We then used logistic regression model with stepwise variable selection procedures to identify risk factors for ASD. Difference was considered statistically significant at $P<0.05$. All statistical analyses were carried out by SAS software, version 8.2 .

\section{Results}

\subsection{General Information of Participants and ASD Prevalence}

In total, 15,417 children were recruited for this study, among whom 7,941 were male and 7,476 were female. There were 7,543 and 7,874 participants in urban and rural area, respectively. Children aged 18-20 months, 21-23 months, 24-29 months, and 30-36 months were 3,319, 3,254, 4,242 and 4,602 , respectively. There was no significant difference in age of diagnosis for ASD $\left(\chi^{2}=0.90, P=0.27\right)$.

As shown in Table 1, all children $(874,5.67 \%$ ) were suspected to be ASD after the Chat-23 screening test. Of these ASD suspected cases, 605 were boys with a positively screening rate of $7.62 \%$ and 269 girls with a positively screening rate of $3.60 \%(P<0.001)$. The positive rates of ASD screening test were similar between urban and rural regions (5.69\% vs. $5.65 \% ; P=0.93)$. There were 429 in 7,543 children $(5.69 \%)$ in rural regions who were suspected to be ASD and 445 in 7874 children (5.65\%) in the urban regions ( $P$ $=0.93$ ).

Table 1. ASD screening and diagnosed results by residence, gender, and age.

\begin{tabular}{llllll} 
& \multirow{2}{*}{$\mathbf{N}$} & \multicolumn{2}{c}{ ASD screening } & \multicolumn{2}{c}{ ASD diagnosis } \\
\cline { 3 - 6 } & & number & ASD prevalence via screening positive rate (\%) & number & ASD diagnosed positive rate (\%) \\
\hline Residence & & & 5.69 & 43 & 0.57 \\
Urban & 7543 & 429 & 5.65 & 46 & 0.58 \\
Rural & 7874 & 445 & & & 43 \\
\hline
\end{tabular}




\begin{tabular}{llllll}
\hline & \multirow{2}{*}{$\mathbf{N}$} & \multicolumn{2}{l}{ ASD screening } & \multicolumn{2}{l}{ ASD diagnosis } \\
\cline { 3 - 6 } & & number & ASD prevalence via screening positive rate (\%) & number & ASD diagnosed positive rate (\%) \\
\hline Gender & & & 3.60 & 16 & 0.21 \\
Female & 7476 & 269 & 7.62 & 73 & 0.92 \\
Male & 7941 & 605 & & & \\
Age & & & 6.48 & 22 & 0.54 \\
18 months ${ }^{+}$ & 3319 & 215 & 6.70 & 25 & 0.68 \\
21 months & 3254 & 218 & 5.21 & 24 & 0.59 \\
24 months & 4242 & 221 & 4.78 & 22 & 0.52 \\
$30-36$ months & 4602 & 220 &
\end{tabular}

Among the 874 children who were positive in the ASD screening test, 89 cases were further confirmed with ASD diagnoses. Overall, the ASD prevalence in children between 18 and 36 months old was $0.58 \%$ in Hubei province. Among 89 children diagnosed with ASD, 73 were males $(0.92 \%)$ and 16 were females $(0.21 \%)$. There was a significantly higher prevalence rate in males than in females $\left(\chi^{2}=19.46, P<0.0001\right)$. There was no significant difference in the prevalence rate of ASD diagnosis between urban and rural area $(0.58 \%$ vs. $0.57 \%$; $P=0.90)$.

\subsection{Comparison of Characteristics of the Participants According to ASD Status}

The univariate results in Table 2 showed that the prevalence of older mother or father, age of delivery, preterm birth, birth weight $<2.0 \mathrm{~kg}$, infection during pregnancy, fetal protection therapy, perinatal asphyxia, pathologic jaundice, hypoxic-ischemic encephalopathy, or gestational diabetes were significantly higher in ASD cases than those without ASD (all $P<0.05$ ).

Table 2. Characteristics of participants by ASD status.

\begin{tabular}{|c|c|c|c|c|}
\hline & Number of ASD & Number without ASD & $\chi^{2}$ & $\boldsymbol{P}$ \\
\hline \multicolumn{5}{|c|}{ Mother age (y) } \\
\hline$<35$ & 19 & 8095 & \multirow{2}{*}{25.63} & \multirow{2}{*}{$<0.0001$} \\
\hline $35+$ & 70 & 7233 & & \\
\hline \multicolumn{5}{|c|}{ Father age (y) } \\
\hline$<35$ & 23 & 9318 & \multirow{2}{*}{22.37} & \multirow{2}{*}{$<0.0001$} \\
\hline $35+$ & 66 & 6010 & & \\
\hline \multicolumn{5}{|l|}{ Sex } \\
\hline Female & 16 & 7460 & \multirow{2}{*}{19.46} & \multirow{2}{*}{$<0.0001$} \\
\hline \multicolumn{3}{|c|}{ Residence } & & \\
\hline Urban & 43 & 7500 & \multirow{2}{*}{0.013} & \multirow{2}{*}{0.91} \\
\hline Rural & 46 & 7828 & & \\
\hline \multicolumn{5}{|c|}{ Preterm birth } \\
\hline Yes & 54 & 2067 & \multirow{2}{*}{42.24} & \multirow{2}{*}{$<0.0001$} \\
\hline No & 35 & 13261 & & \\
\hline \multicolumn{5}{|c|}{ Birth weight (kg) } \\
\hline $2.0+$ & 58 & 14811 & 28.34 & $<0.0001$ \\
\hline \multicolumn{5}{|c|}{ Infection during pregnancy } \\
\hline Yes & 65 & 2067 & \multirow{2}{*}{63.93} & \multirow{2}{*}{$<0.0001$} \\
\hline No & 24 & 13261 & & \\
\hline \multicolumn{5}{|c|}{ Fetus protection therapy } \\
\hline Yes & 63 & 1955 & \multirow{2}{*}{37.20} & \multirow{2}{*}{$<0.0001$} \\
\hline No & 26 & 13373 & & \\
\hline \multicolumn{5}{|c|}{ Perinatal asphyxia } \\
\hline Yes & 74 & 2756 & \multirow{2}{*}{75.18} & \multirow{2}{*}{$<0.0001$} \\
\hline No & 15 & 12572 & & \\
\hline \multicolumn{5}{|c|}{ Pathologic jaundice } \\
\hline Yes & 69 & 1206 & & \\
\hline No & 20 & 14122 & 87.77 & $<0.0001$ \\
\hline Hypoxic & & & & \\
\hline Yes & 55 & 1442 & 5133 & $<00001$ \\
\hline Yes & 23 & 1894 & & \\
\hline No & 66 & 13434 & 5.21 & 0.02 \\
\hline
\end{tabular}

\subsection{Multivariate Logistic Regression of Risk Factors for ASD}

As exhibited in Table 2, multivariate logistic regression was carried out with the significant risk factors from univariate analyses. Stepwise selection procedure was applied. It indicated that mothers or fathers 35 years or older, preterm birth, and birth weight $<2.0 \mathrm{~kg}$ remained significantly associated with higher risk of ASD (Table 3). 
Table 3. Multivariable-adjusted ORs and 95\% CIs of ASD of its risk factors.

\begin{tabular}{lll}
\hline Risk factors & ORs $(\mathbf{9 5 \%}$ CI) & P value \\
\hline Mother age 35+ & $1.57(1.17-2.58)$ & $<0.0001$ \\
Father age 35+ & $1.61(1.27-2.99)$ & $<0.0001$ \\
Preterm & $1.87(1.62-2.16)$ & $<0.0001$ \\
Birth weight $<2.0 \mathrm{~kg}$ & $1.24(1.05-1.46)$ & 0.01 \\
\hline
\end{tabular}

\section{Discussion}

This population-based survey data showed that the overall prevalence of autism was estimated at 5.8 per 1000 among children aged 18 to 36 months living in Hubei, China. The ASD prevalence in male and female were 9.2 per 1000 and 2.1 per 1000 , respectively. We also found that older mother or father, age of delivery, preterm birth, and birth weight $<2.0 \mathrm{~kg}$ was significantly associated with higher risk of ASD.

\subsection{Prevalence of ASD}

In the United States, the overall estimated prevalence of ASD among the 14 sites of the Autism and Developmental Disabilities Monitoring (ADDM) was 11.3 per 1000 children who were around eight years old [4]. On April 26, 2018, the Centers for Disease Control and Prevention (CDC) released new data on the prevalence of autism in the United States. This surveillance study identified 1 out of 59 children (approximately 17 per 1,000 ) as ASD patients. In 2011, the prevalence of autism was estimated at 4.1 per 1000 in Australia [12]. Almost one-third of ASD cases were related to high function ASD [3].

On the other hand, ASD survey data from large-scale population-based epidemiological studies in China had not been reported. We did thorough survey on prevalence of ASD at China National Knowledge Infrastructure (CNKI), Wanfang database and PubMed database between 2000-2016, and identified 21 papers published in 15 journals about the epidemiology of ASD among children in China. We estimated the pooled prevalence of ASD is $0.24 \%$ (2.4 per 1000), which is generally lower than those reported in the present study and most of western countries [13]. Of note, the prevalence of ASD was similar between urban area (1.7 per 1000) and rural area (0.9 per 1000) [13].

In addition, ASD diagnosis criteria have a high degree of heterogeneity in previous studies. The prevalence of ASD may vary due to different screening and diagnostic methods. The present study among a representative sample of Chinese children used the CHAT-23 for screening and the childhood autism rating scale (CARS) and the DSM-5 for further diagnosis. In this paper, the reported ratio of $0.58 \%$ for ASD prevalence is reasonable among Chinese children of 18 to 36 months.

\subsection{Gender Differences in the Prevalence of ASD}

ASD shows a striking gender difference, with approximately $4: 1$ of male to female ratio [14]. In 2018, the combined estimated prevalence of ASD among 11 ADDM Network sites was 1 in 42 boys (24 per 1,000) and 1 in 189 girls (5 per 1,000) (https://www.autismspeaks.org/science-news/cdc-increases-esti mate-autisms-prevalence-15-percent-1-59-children). Estimated prevalence was significantly higher among boys aged 8 (23.6 per $1,000)$ than girls aged $8(5.3$ per 1,000$)[15]$. Our study further confirmed this gender difference of ASD prevalence in Chinese children, though the male to female ratio was slightly different. Of 89 confirmed ASD cases aged from 18 to 36 months, there were 73 males $(9.2$ per 1,000$)$ and 16 females $(2.1$ per 1,000$)$, and the ratio of male to female is 4.38 to 1 . As we reviewed the literature of ASD prevalence in China from 21 papers published between 2000 and 2016, we found similarly gender-specific difference in the ASD prevalence in Chinese children, which were 3.5 per 1000 for male and 0.7 per 1000 for female [13].

However, mechanisms underlying sexually dimorphic ASD prevalence are still poorly understood. Biological explanations for the sex difference in ASD prevalence were most relevant to genetic basis [16]. It has been hypothesized that females with ASD are likely to carry a higher heritable mutational "load" than affected males [17]. Evidences suggested that sex chromosomal genes and/or sex hormones, especially testosterone, may modulate the effects of genetic variation on the presentation of an autistic phenotype [16]. Recent genetic studies have suggested that females may be protected from the effects of heritable and de novo ASD risk variants. For instance, recent studies have identified the first inherited autosomal variant, a rare copy number variants (CNVs) in SHANK1 gene, which encodes scaffolding proteins at the postsynaptic site of excitatory synapses in the brain [18, 19] Males carrying this variant had high-functioning autism while female relatives carrying the same variant showed anxiety, indicating a clearly reduced penetrance in female [20]. While they cannot fully explain the gender difference, females with ASD tend to have fewer restricted and repetitive behaviors and externalizing behavioral problems than males.

\subsection{Regional Differences in the Prevalence of ASD}

The prevalence of ASD may differ in the demography, including racial/ethnic, and socioeconomic groups or health care services. The latter includes diagnostic and developmental evaluations. Estimated prevalence varied widely among the 11 ADDM Network sites, ranging from 8.2 per 1,000 children to 24.6 per 1,000 children [2]. Estimated ASD prevalence was significantly higher among Caucasian children (15.5 per 1,000) than among black children (13.2 per $1,000)$, and Hispanic $(10.1$ per 1,000$)$ children $[1,2,21]$

Although previous studies indicated that prevalence of ASD in the urban areas of China was significantly higher than that in rural areas, it is still too early to make a conclusion that regions make difference. It is possible that these differences may be associated with ASD recognition and awareness in specialist and/or the parents, research methods and availability of diagnostic services. Our study showed no statistically significant differences in the prevalence rate of ASD between urban and rural areas. This study has been equipped with various measurement standards and strict quality control, in order to avoid subjective bias and advance our understanding of the impact of early diagnosis. 


\subsection{Risk Factors of ASD}

The dramatic increase in ASD patients in recent years cannot be solely explained by the broadening of diagnostic criteria. Our study showed that mothers or fathers aged 35 years or older, preterm birth, and birth weight $<2.0 \mathrm{~kg}$ were significantly and independently associated with risk of ASD. When a baby is conceived, a father's and /or mother's age is the single largest factor in the risk of passing on new gene mutations to children [22]. The age-linked increase in mutations proportionally increased the possibility whether a child might carry a harmful mutation related to ASD [23].

Although there is a strong genetic contribution to autism, it is clear that environmental factors such as maternal infection can also increase the risk for this disorder [24]. Our study showed that infection during pregnancy was associated with increased risk of ASD. Maternal infection may alter fetal brain development to cause these abnormalities [10]. Activation of mother's immune system increases the level of one protein (cytokine), termed IL-6, which is important to fight against infection. Moreover, this elevation of IL-6 alters the function of the placenta that is critical for the development of autism-like symptoms in the offspring [25]. Our study suggests that low birth weight (LBW) and premature infants are associated with increased risk of ASD. The association between ASD and LBW might be mediated by several prenatal or neonatal factors, such as maternal age and health conditions, nutrition deficits, hypoxia, and other obstetrical factors that were described in association with prematurity and LBW [26]. Our previous study found that the incidence of preterm birth was positively associated with maternal age 35 or higher.

Murray and Harvey reported that brains of individuals with autism have also been shown morphological hippocampal abnormalities [27]. This study suggests that prenatal, perinatal and neonatal conditions associated with fetal hypoxia are likely to be heterogeneous in origin and may include premature birth, asphyxia, hypoxic-ischemic encephalopathy, and pathologic jaundice. Early detection of brain structural alterations may allow the early identification and intervention of children at risk of ASD [28].

\subsection{Strength and Limits}

The major strength of this study is the use of representative sample of Chinese children aged 18 to 36 months, which has provided the opportunity to provide a true prevalence value of Chinese children with relatively homogenous genetic and lifestyle backgrounds. Also, a standard pediatrician training procedure was performed at each site of the local hospitals and the Hubei Maternity and Child Health Care Hospital to ensure the appropriate use of screen and diagnostic tools for ASD. Certainly, several limits of this study need to be acknowledged. First, the relatively small sample size of ASD cases, comparing to the huge population in China, has limited our ability to fully address sex and geography-specific differences. Secondly, lack of biomarker and genetic information on ASD cases has hindered understanding of ASD etiology or gender-different prevalence from the perspective of mechanism. Thirdly, due to lack of complete and accurate data on all potential confounders and plausible mediators, ASD risk factors cannot be comprehensively evaluated in this study. In addition, this is a cross-sectional study, which did not allow us to infer any causal relationships between risk factors and ASD development.

\section{Conclusion}

In conclusion, our screening study suggested an ASD prevalence of $5.67 \%$ with approximately $4: 1$ of male: female ratio based on a representative sample of 15,417 children 18 to 36 months living in Hubei, China. Clinical diagnosis further narrowed down the prevalence rate of ASD to around $0.58 \%$. There is no significant difference in the ASD risk between urban and rural regions. We also found that mothers or fathers aged 35 years or older, preterm birth, and birth weight $<2.0 \mathrm{~kg}$ were significantly and independently associated with risk of ASD. For future studies, large, population-based birth cohorts will be needed to allow for precise and detailed assessments of exposures and potential confounders.

\section{Author Contributions}

Qiong Dai and Xuejun Kong contributed equally to this work. They wrote the initial draft and revised it. Yiqing Song, Aiqin Zhou, Xuan Zhang, Ping Zhang, Minghui Li and Xinglian Liu collected the data and analyzed. Jun Wang and Haiqing Xu were the project leadership and designed the work.

\section{Conflicts of Interest Statement}

The authors report no conflict of interest.

\section{Acknowledgements}

We thank to Natural Science Foundation of Hubei Province, for financial support (Grant number: 1020006301).

\section{References}

[1] Coo H, Ouellette-Kuntz H, Lloyd JE, Kasmara L, Holden JJ, et al. Trends in autism prevalence: diagnostic substitution revisited. J Autism Dev Disord. 2008; 38 (6): 1036-1046.

[2] Wingate M, Kirby RS, Pettygrove S, Cunniff C, Schulz E, et al. Prevalence of autism spectrum disorder among children aged 8 years - autism and developmental disabilities monitoring network, 11 sites, United States, 2010. MMWR Surveill Summ. 2014; 63 (2): 1-21.

[3] Baird G, Simonoff E, Pickles A, Chandler S, Loucas T, et al. Prevalence of disorders of the autism spectrum in a population cohort of children in South Thames: the Special Needs and Autism Project (SNAP). Lancet. 2006; 368 (9531): 210-215.

[4] Mulvihill B, Wingate M, Kirby RS, Pettygrove S, Cunniff C, et al. Prevalence of Autism Spectrum Disorders - Autism and Developmental Disabilities Monitoring Network, United States, 2006. MMWR Surveill Summ. 2009; 58 (10): 1-20. 
[5] Skonieczna-Zydecka K, Gorzkowska I, Pierzak-Sominka J, Adler G. The Prevalence of Autism Spectrum Disorders in West Pomeranian and Pomeranian Regions of Poland. J Appl Res Intellect Disabil. 2016; 30 (2): 283-289.

[6] Zachor D, Yang JW, Itzchak EB, Furniss F, Pegg E, et al. Cross-cultural differences in comorbid symptoms of children with autism spectrum disorders: an international examination between Israel, South Korea, the United Kingdom and the United States of America.. Dev Neurorehabil. 2011; 14 (4): 215-220.

[7] Dobson S, Upadhyaya S, McNeil J, Venkateswaran S, Gilderdale D. Developing an information pack for the Asian carers of people with autism spectrum disorders. Int J Lang Commun Disord. 2001; 36 Suppl: 216-221.

[8] Elsabbagh M, Divan G, Koh YJ, Kim YS, Kauchali S, et al. Global prevalence of autism and other pervasive developmental disorders. Autism Res. 2012; 5 (3): 160-179.

[9] Risi S, Lord C, Gotham K, Corsello C, Chrysler C, et al. Combining information from multiple sources in the diagnosis of autism spectrum disorders. J Am Acad Child Adolesc Psychiatry. 2006; 45 (9): 1094-1103.

[10] Larsson HJ, Eaton WW, Madsen KM, Vestergaard M, Olesen $\mathrm{AV}$, et al. Risk factors for autism: perinatal factors, parental psychiatric history, and socioeconomic status. Am J Epidemiol 2005; 161 (10): 916-925.

[11] Yassin W, Kojima M, Owada K, Kuwabara H, Gonoi W, et al. Paternal age contribution to brain white matter aberrations in autism spectrum disorder. Psychiatry Clin Neurosci. 2019; 73 (10): 649-659.

[12] Taylor LJ, Eapen V, Maybery MT, Midford S, Paynter J, et al. Diagnostic evaluation for autism spectrum disorder: a survey of health professionals in Australia. BMJ Open. 2016; 6 (9): e012517.

[13] Dai Q, Xu HQ. Analysis of Bibliometric Papers on the epidemiology of Children with Autism Spectrum Disorders in China form 2000-2016 years. Chinese Journal of Child Health Care.2017; (25): 271-274.

[14] Lyall K, Croen L, Daniels J, Fallin MD, Ladd-Acosta C, et al. The Changing Epidemiology of Autism Spectrum Disorders. Annu Rev Public Health. 2017; 38: 81-102.

[15] Mintz, ME. A parent-centered approach to autism diagnosis in early childhood. World J Pediatr. 2018; 14 (3): 212-214.

[16] Carter AS, Black DO, Tewani S, Connolly CE, Kadlec MB, et al. Sex differences in toddlers with autism spectrum disorders. J Autism Dev Disord. 2007; 37 (1): 86-97.

[17] Rai D, Lee BK, Dalman C, Golding J, Lewis G, et al. Parental depression, maternal antidepressant use during pregnancy, and risk of autism spectrum disorders: population based case-control study. BMJ. 2013; 346: f2059.

[18] Leblond CS, Nava C, Polge A, Gauthier J, Huguet G, et al. Meta-analysis of SHANK Mutations in Autism Spectrum Disorders: a gradient of severity in cognitive impairments. PLoS Genet. 2014; 10 (9): e1004580.

[19] Gong X, Wang H. SHANK1 and autism spectrum disorders. Sci China Life Sci. 2015; 58 (10): 985-990.

[20] Sato D, Lionel AC, Leblond CS, Prasad A, Pinto D, et al. SHANK1 Deletions in Males with Autism Spectrum Disorder. Am J Hum Genet. 2012; 90 (5): 879-887.

[21] Chakrabarti S, Fombonne E. Pervasive developmental disorders in preschool children: confirmation of high prevalence. Am J Psychiatry. 2005; 162 (6): 1133-1141.

[22] Manzouri L, Yousefian S, Keshtkari A, Hashemi N. Advanced Parental Age and Risk of Positive Autism Spectrum Disorders Screening. Int J Prev Med. 2019; 10: 135.

[23] Shelton JF, Hertz-Picciotto I, Pessah IN. Tipping the balance of autism risk: potential mechanisms linking pesticides and autism. Environ Health Perspect. 2012; 120 (7): 944-951.

[24] Tran PL, Lehti V, Lampi KM, Helenius H, Suominen A, et al., Smoking during pregnancy and risk of autism spectrum disorder in a Finnish National Birth Cohort. Paediatr Perinat Epidemiol. 2013; 27 (3): 266-274.

[25] Smith SE, Li J, Garbett K, Mirnics K, Patterson PH. Maternal immune activation alters fetal brain development through interleukin-6. J Neurosci. 2007; 27 (40): 10695-10702.

[26] Pinto D, Delaby E, Merico D, Barbosa M, Merikangas A, et al. Convergence of Genes and Cellular Pathways Dysregulated in Autism Spectrum Disorders. Am J Hum Genet. 2014; 94 (5): 677-694.

[27] Diehl JJ, Schmitt LM, Villano M, Crowell CR. The clinical use of robots for individuals with Autism Spectrum Disorders: A critical review. Res Autism Spectr Disord. 2012; 6 (1): 249-262.

[28] Padilla N, Eklöf E, Mårtensson GE, Bölte S, Lagercrantz H, et al. Poor Brain Growth in Extremely Preterm Neonates Long Before the Onset of Autism Spectrum Disorder Symptoms. Cereb Cortex. 2017; 27 (2): 1245-1252. 